\title{
Development of Robotic Vision Algorithm for Medical Application
}

\author{
Reena Mercy S. ${ }^{1}$, Mrs. Parnasree Chakraborty ${ }^{2}$ \\ ${ }^{1,}$ M. Tech Student, II Year, Department of Communication Systems, \\ ${ }^{2}$ Assistant Prof., Department Of Communication Systems, B.S. AbdurRahman University Vandalur, Chennai
}

\begin{abstract}
Today's world is of high-tech and improved facilities. Especially in the field of medicine, advanced technologies have been implemented. One of the feature includes automatic work aiding using robotics. This system deals with reducing duty workload in clinical operations. Currently, the robots are not developed at the level where they can function like human in medical and health care professionals. Currently nursing robots are used in many countries. It only used for clinical purposes like medicine distributions. In this system, some additional features are to be included. Which means the robot is highly intelligent and efficient. One of the features is motion detection, to monitor the movements of the patient. It detects excessive bleeding of patient, and monitors the heartrate by reconfiguring the machine learning algorithm and also added special angle mapping identification of object using digital image processing.
\end{abstract}

Keyword: Reconfigured machine learning algorithm, nursing robots, motion detection, bleeding.

\section{A. Robotics}

\section{Introduction}

A robot can be defined as a programmable, self-controlled device consisting of electronic, electrical, or mechanical units. More generally, it is a machine that functions in place of a living agent. Robots are especially desirable for certain work functions because, unlike humans, they never get tired. They can endure physical conditions that are uncomfortable or even dangerous. They can operate in airless conditions; they do not get bored by repetition; and they cannot be distracted from the task at hand. A humanoid robot is a robot with its body shape built to resemble that of the human body. A humanoid design might be for functional purposes, such as interacting with human tools and environments, for experimental purposes, such as the study of bipedal locomotion, or for other purposes. In general, humanoid robots have a torso, a head, two arms, and two legs; though some forms of humanoid robots may model only part of the body, for example, from the waist up. Some humanoid robots may also have heads designed to replicate human facial features such as eyes and mouths. Androids are humanoid robots built to aesthetically resemble humans

\section{B. Robotic Nurse Assistant}

There is a well-documented shortage of nurses and direct-care workers in the U.S. and around the world, which is expected to become more problematic as the older adult population grows and prepares for retirement. In a study of the effects of high patient-to-nurse ratio, Aiken et al. showed that each additional patient per nurse was associated with a $7 \%$ increase in patient mortality and a $23 \%$ increase in nurse burnout. Consequently, studies have suggested that lowering the patient-to-nurse ratio would result in less missed patient care. We believe robotics can play a role in assisting nurses to complete their daily tasks in order to provide better healthcare. Itincludes some features such asmotion detection,heart ratemonitoring, bleeding detection, detecting toxic gases and also having sensor for monitoring the humidity of the room with some physical changes and the GUI is developed for ease access.

\section{Motion Detection}

Motion detection is the process of detecting a change in position of an object relative to its surroundings or the change in the surroundings relative to an object. Motion detection can be achieved by both mechanical and electronic methods. When motion detection is accomplished by natural organisms, it is called motion perception.

\section{Motion can be detected by:}

1. Infrared (Passive and active sensors).

2. Optics (video and camera systems)

3. Radio Frequency Energy (radar, microwave and tomographic motion detection).

4. Sound (microphones and acoustic sensors).

5. Vibration (triboelectric, seismic, and inertia-switch sensors). 
6. Magnetism (magnetic sensors and magnetometers).

\section{A. Goals of motion detection:}

1. Identify moving objects.

2. Detection of unusual activity patterns.

3. Computing trajectories of moving objects.

\section{B. Optical Flow}

1. Compute motion within region or the frame as a whole

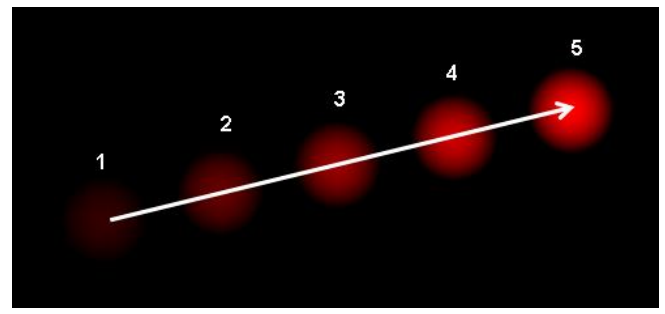

Fig.1. Principle of motion detection

\section{Change detection}

1. Detect objects within a scene

2. Track object across a number of frames

\section{Background Subtraction}

1. Uses a reference background image for comparison purposes.

2. Current image (containing target object) is compared to reference image pixel by pixel.

3. Places where there are differences are detected and classified as moving objects.

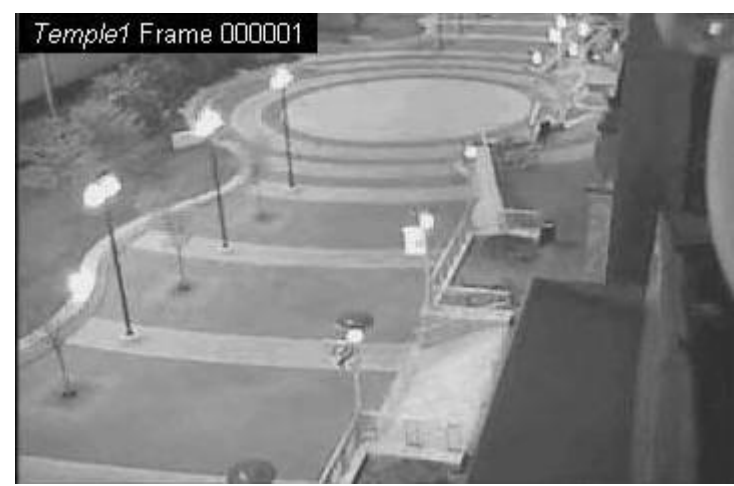

Fig.2. Previous image

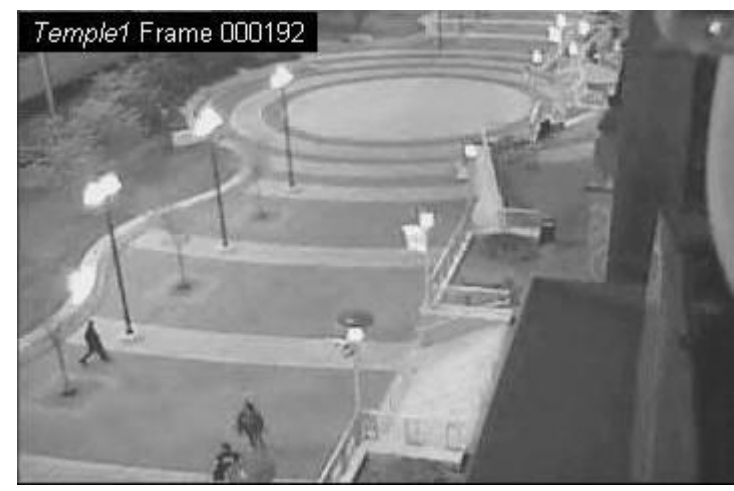

Fig.3. Current image 


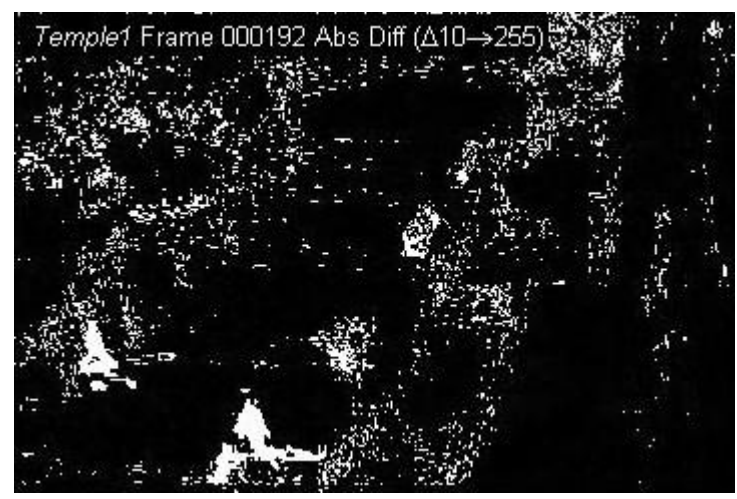

Fig.4. Binary image difference

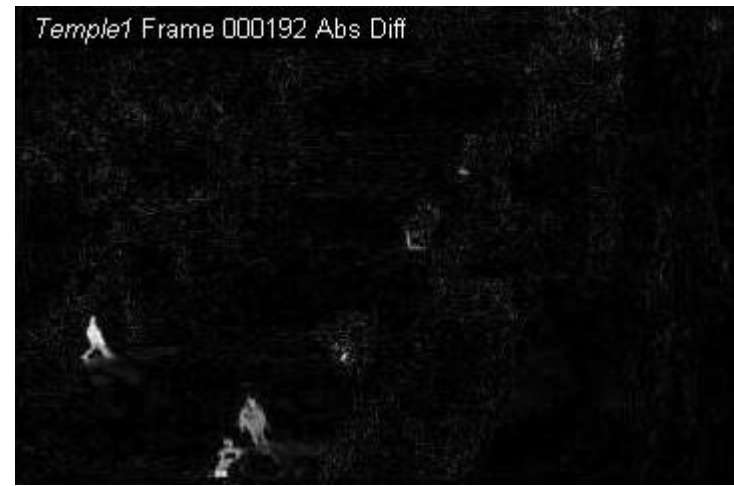

Fig 5. Motion detection

A simple algorithm for motion detection by a fixed camera compares the current image with a reference image and simply counts the number of different pixels. Since images will naturally differ due to factors such as varying lighting, camera flicker, and CCDdark currents, pre-processing is useful to reduce the number of false positivealarms. More complex algorithms are necessary to detect motion when the camera itself is moving, or when the motion of a specific object must be detected in a field containing other movement which can be ignored. An example might be a painting surrounded by visitors in an art gallery.

\section{E. Threshold}

A greyscale image is turned into a binary image by first choosing a gray level $\mathrm{T}$ in the original image, and then turning every pixel black or white according to whether its gray value is greater than or less than T. A pixel becomes white if its gray level is $>\mathrm{T}$. A pixel becomes black if its gray level is $<=\mathrm{T}$.

\section{F. ALGORITHM}

Step1: video input is taken as number of frames per interval.

Step2: the frames are compared with the previous one simultaneously.

Step3: if there is any difference between the frames means then there will be a movement occurs.

Step4: if motion is detected means it will display. 


\section{G.FLOWCHART}

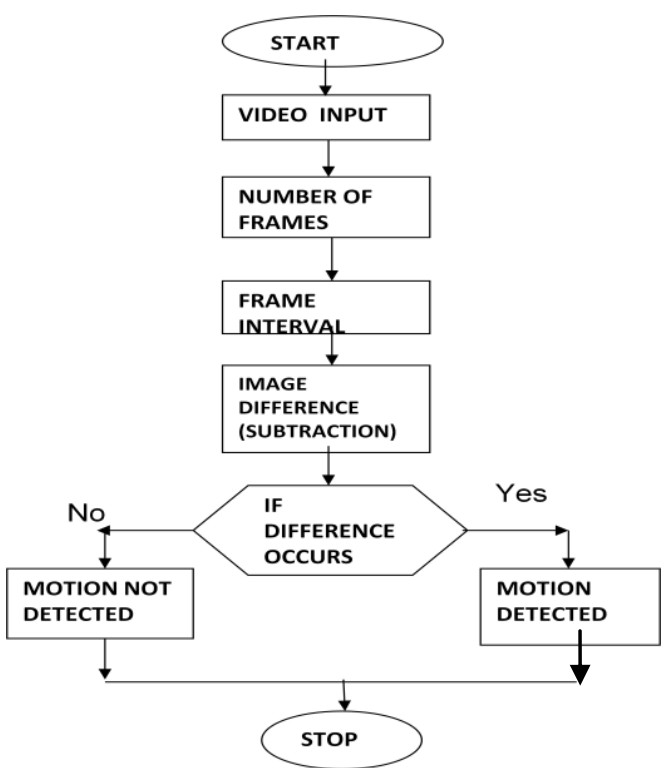

Fig. 6. Flow chart

\section{Blood Detection}

In order to find out the bleeding of patient, a camera will be fixed into the robot. This is used to monitor the patient. By using image processing in mat lab it can be possible to identify where the bleeding is occurring.

\section{A. Description:}

Basic idea behind this project is: image processing robots are used to detect whether a patient is injured or bleeding. This can be done by image processing colour detection and threshold allocating method.

This project mainly deals with colour image processing and this chapter first presents the fundamentals of colour followed by a discussion of several commonly used colour models. Next, several examples of using colour image processing will be given, including the correction of the spatial filtering, the detection of edges, the histogram equalization, the colour white balancing of colour images, edge detection and motion detection. The final section discusses a technique known as threshold used to highlight specific gray levels in a gray scale image in colour to enhance important features so that they are clearly observable.

\section{B. Colour Descriptors:}

Colour is important in image processing robot because colour is a powerful descriptor that simplifies object identification and extraction. Humans can discern thousands of colour shades and intensities, compared to about only two dozen shades of gray.

Colour image processing is divided into two major areas:

1. Full-colour processing: images are acquired with a full-colour sensor, such as colour TV camera or colour scanner.

2. Pseudo colour processing: The problem is one of assigning a colour to a particular monochrome intensity or range of intensities.

\section{Colour Fundamentals:}

Colours are seen as variable combinations of the primary colours of light red $(\mathrm{R})$, green $(\mathrm{G})$, and blue (B). The primary colours can be mixed to produce the secondary colours: magenta (red + blue), cyan (green + blue), and Yellow (red + green). Mixing the three primaries, or a secondary with its opposite primary colour, produces white light. RGB colours are used for colour TV, monitors, and video cameras. However, the primary colours of pigments are cyan (C), magenta (M), and yellow (Y), and the secondary colours are red, green, and blue. A proper combination of the three pigment primaries, or a secondary with its opposite primary, produces black. 

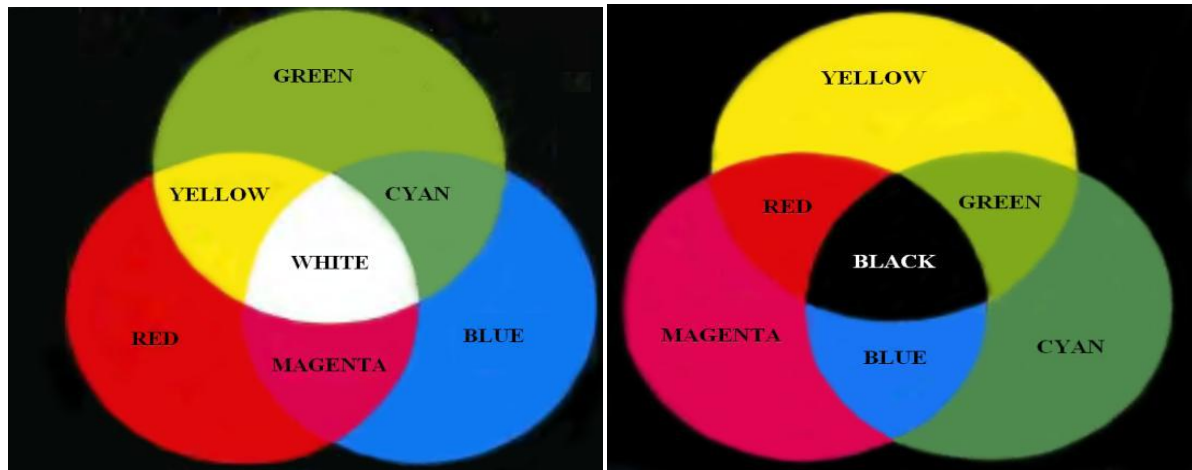

Fig. 7. Primary and secondary colours

\section{Development Of Robotic Vision Algorithm For Medical Application}

The purpose of a colour model is to facilitate the specification of colours in some standard way. A colour model is a specification of a coordinate system and a subspace within that system where each colour is represented by a single point.

Colour models most commonly used in image processing are:

1. RGB model for colour monitors and video cameras

2. CMY and CMYK (cyan, magenta, yellow, black) models for colour printing

3. HSI (hue, saturation, intensity) model

In this project, we use the RGB colour model only let us briefly see about that

\section{E.The RGB colour model}

In this model, each colour appears in its primary colours red, green, and blue. This model is based on a Cartesian coordinate system. All colour values R, G, and B have been normalized in the range [0, 1]. However, we can represent each of R, G, and B from 0 to 255. Each RGB colour image consists of three component images. RGB system, each colour pixel can be expressed as For an image of size $\mathrm{M} \times \mathrm{N}$, there are MN such vectors, $\mathrm{c}(\mathrm{x}, \mathrm{y})$, for $\mathrm{x}=0,1,2, \ldots, \mathrm{M}-1 ; \mathrm{y}=0,1,2, \ldots, \mathrm{N}-1$.

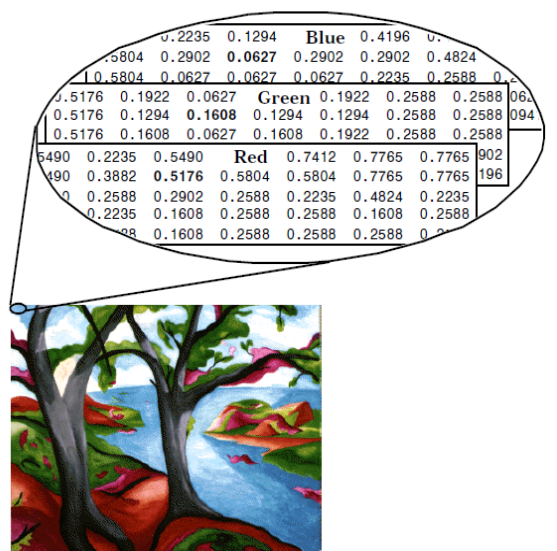

Fig.8. True RGB colour model

\section{F. ALGORITHM}

Step1:Taking the video input as number of frames per interval.

Step2:The frames are compared with the previous one simultaneously in RGB format.

Step3:If it founds any red colored object means it will indicate with the location also. 


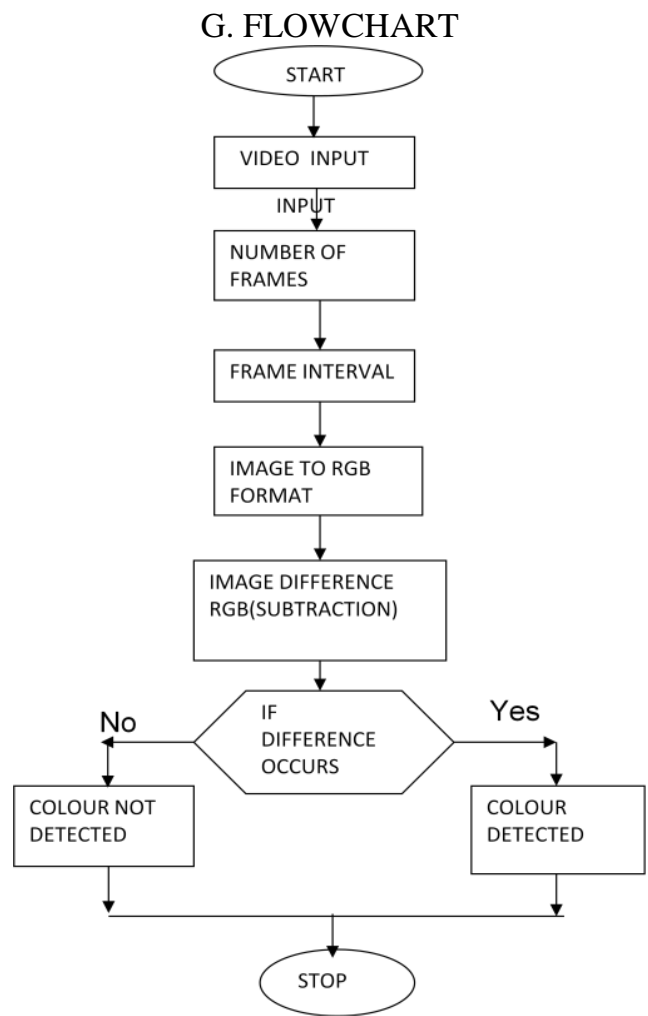

Fig.9. Flowchart

\section{Heart Rate Monitoring}

To measure the heart rate of the patient a heartbeat sensor will be fixed into the robot. It will find out that the heart rate is in normal condition or whether it is in abnormal condition.

\section{A. HEARTBEAT SENSOR}

Heart beat sensor is designed to give digital output of heat beat when a finger is placed on it. When the heart beat detector is working, the beat LED flashes in unison with each heart beat. This digital output can be connected to microcontroller directly to measure the Beats Per Minute (BPM) rate. It works on the principle of light modulation by blood flow through finger at each pulse.

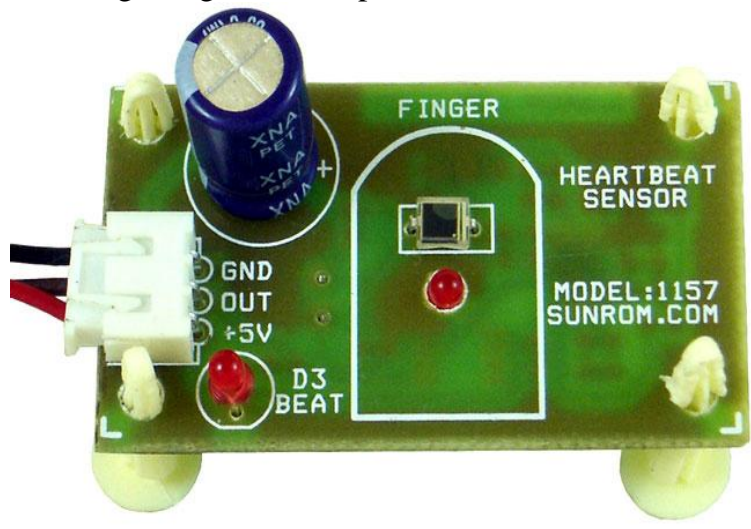

Fig.10.Heartbeat sensor

\section{B. Features}

The features of the sensor are Heat beat indication by LED, Instant output digital signal for directly connecting to microcontroller, Compact Size and having Working Voltage as +5V DC. 


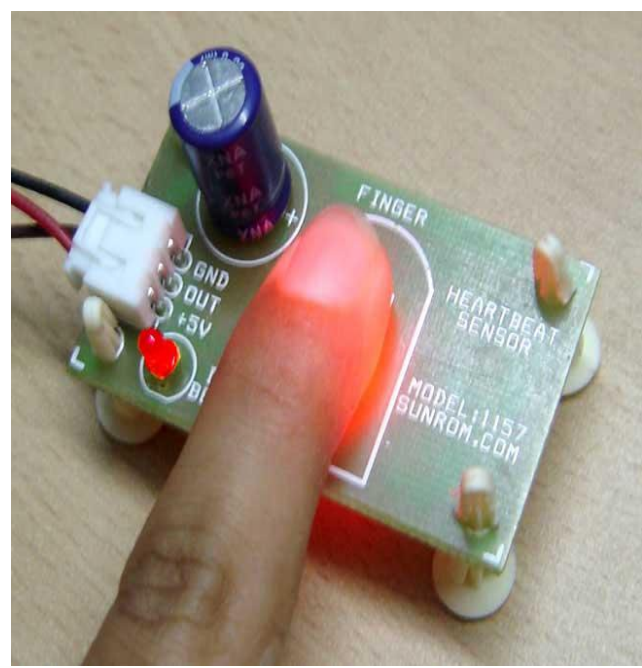

Fig.11.Using the sensor

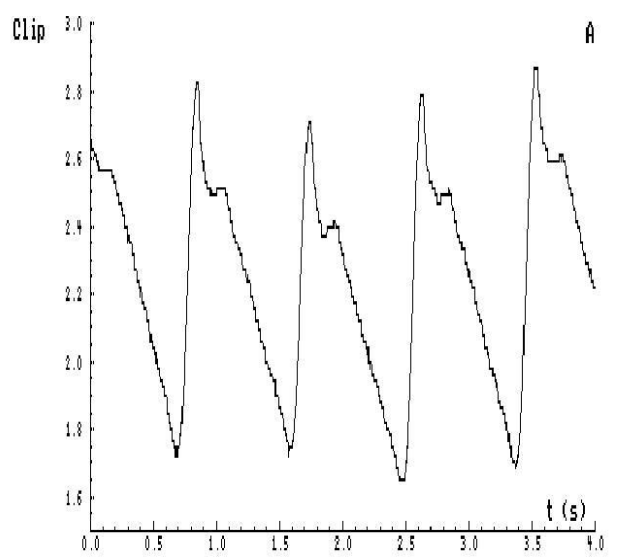

Fig. 12.Heartbeat output signal

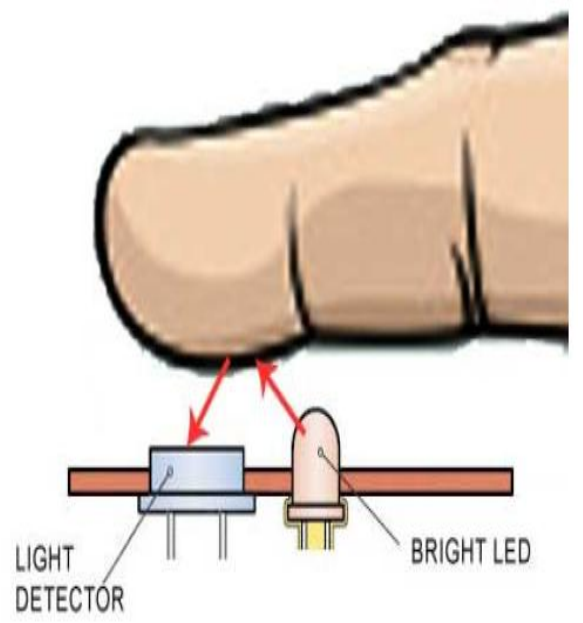

Fig.13.Sensor principle 
1. MOTION DETECTION

\section{Simulation Results}

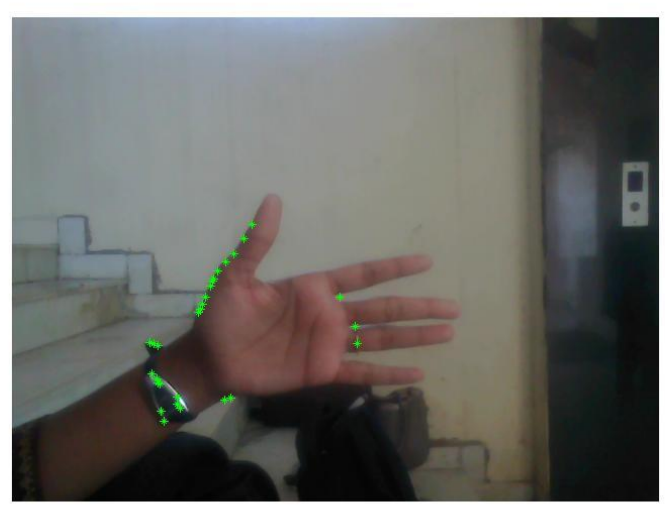

Fig. 14. Motion detection

The fig 6 states about motion detection. From the above figure the green colour dots indicate that the hand is moving.

\section{BINARY IMAGE}

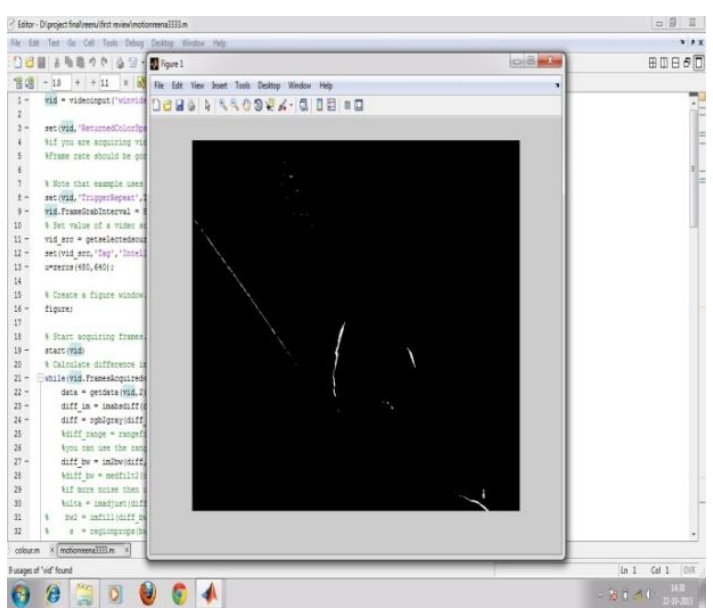

Fig.15(a). Binary image

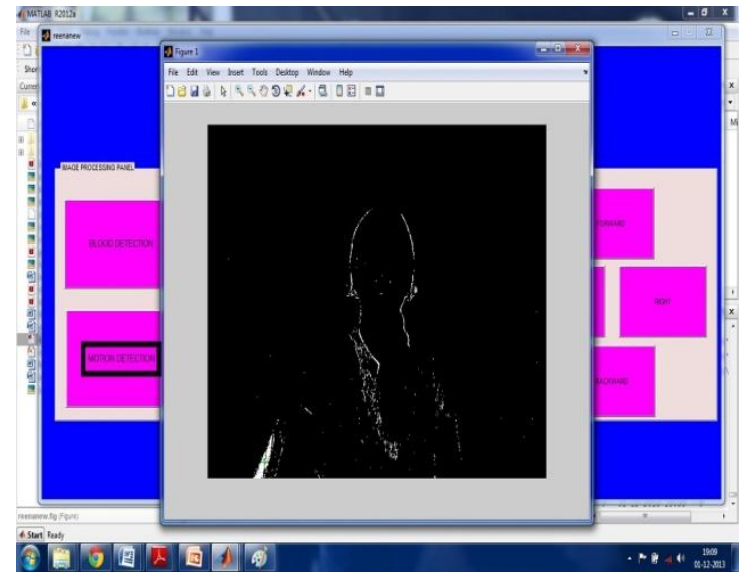

Fig.15(b). Binary image in gui 


\section{BLOOD DETECTION}

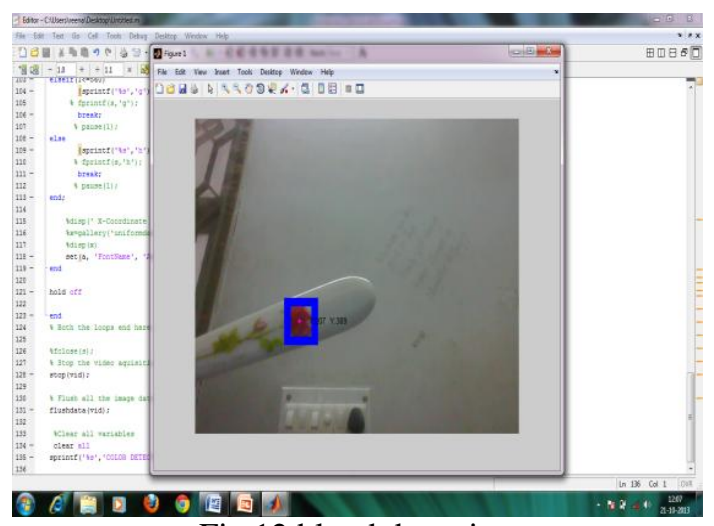

Fig.12 blood detection

\section{Conclusion}

This Nursing Robot at monitoring of patient and emergency is also likely to be more robust. It detects the bleeding with the blood colour detection and movement of the patient, using image processing system and detects patient's movement which helps them by providing first aid kit to them and also controlled by patients. This will help the patient if any emergency. The heartbeat of the patient also monitors using the sensor. In future we can implement this idea in humanoid with additional features.

\section{Reference}

[1] Cyberbotics. Webots: Professional mobile robot simulation. International Journal of Advanced Robotic Systems, 1(1):39-42, 2004.

[2] K. Fujiwara, Kanehiro F., S. Kajita, K. Yokoi, H. Saito, K. Harada, K. Kaneko, and H. Hirukawa. The first human-size humanoid that can fall over safely and stand-up again. In IEEE/RSJ International Conference on Intelligent Robots and Systems (IROS), pages 1920-1916, 2003, Las Vegas, NV, USA.

[3] P.-B. Wieber. On the stability of walking systems. In Proceedings of the International Humanoid and Human Friendly Robots, 2002.

[4] R. M. Murray, Z. Li, and S. S. Sastry. A Mathematical Introduction to Robotic manipulation. CRC Press, Boca Raton, 1994.

[5] K. Ogata, K. Terada, and Y. Kuniyoshi. Real-time selection and generation of fall damagae reduction actions for humanoid robots. In Humanoids08, pages 233-238, Dec. -3 2008, Daejeon, Korea.

[6] K. Ogata, K. Terada, and Y. Kuniyoshi. Falling motion control for humanoid robots while walking. In IEEE-RAS 7th International Conference on Humanoid Robots, Pittsburgh, 2007.

[7] J. Pratt, J. Carff, S. Drakunov, and A. Goswami. Capture point: A step toward humanoid push recovery. In Humanoids06, December, Genoa, Italy 2006.

[8] M. W. Spong, P. Corke, and R. Lozano. Nonlinear control of inertia wheel pendulum. Automatica, 37:1845-1851, February 2001

[9] Robotic nurse assistant to ferri patients around hospital wards,2009.

[10] Robot assistant will help caregivers, nurses by Robert Janis,2010 